WINPEC Working Paper Series No.E1808

November 2018

Monotonic Core Allocation Paths for Assignment Games

Takaaki Abe and Shuige Liu

Waseda INstitute of Political EConomy

Waseda University

Tokyo, Japan 


\title{
Monotonic Core Allocation Paths for Assignment Games*
}

\author{
Takaaki Abe ${ }^{\dagger}$ and Shuige Liu ${ }^{\ddagger}$
}

September 25, 2018

\begin{abstract}
We introduce a modification of Sprumont [9]'s population monotonic allocation scheme (PMAS), called monotonic core allocation path (MCAP) for assignment games, which is a sequence of allocations along an order on the set of players satisfying that (1) each allocation is in the core of the subgame of the corresponding players at that step, and (2) the payoffs for each player are non-decreasing through the sequence. The notion of MCAP preserves the population monotonicity of PMAS while avoids the difficulty that PMAS does not exist in many market games. We show that a MCAP exists for every assignment game. The terminals of MCAP form a refinement of the core. We also show that the terminals of MCAP coincide with the extreme core allocations in two subclasses of assignment games: gloves games and Böhm-Bawerk games. The strong connection of MCAP with extreme core allocations suggests some conflict between the stability of a coalition formation process and the fairness of the resulting outcomes.
\end{abstract}

Keywords: Allocation path; Assignment game; Core; Population monotonicity

\section{Introduction}

Coalition formation process is an important issue in game theory and economics. Within the framework of cooperative game theory which studies appropriate allocations among players based on payoffs attainable via coalitions, a notion called the core provides a rudimentary yet compelling approach: an allocation is in the core if there are no players who can reject the allocation and attain higher payoffs by forming a coalition of themselves. A core allocation makes a grand coalition preservable, while it does not show how the coalition is formed. Indeed, if coalition formation is taken as a process arising from a small group and growing larger, a core allocation may not be practical since it is not necessarily attainable in every smaller coalition.

A seminal attempt by Sprumont [9] tries to solve this problem by introducing a notion called population monotonic allocation scheme (PMAS). A PMAS is a vector assigning each coalition $S$ an allocation $x^{S}$ such that (1) $x^{S}$ is attainable via $S$, and (2) for coalitions $S, T$ with $S \subseteq T$, the payoff $x_{i}^{S}$ for each player $i \in S$ is at least as much as $x_{i}^{T}$. The second condition is called population monotonicity in the literature of bargaining (Thomson [10], [11]). As Sprumont [9] put it, his concern is to guarantee that once a coalition $S$ has decided upon an allocation attainable via $S$, "no player will ever be tempted to induce the formation of a coalition smaller than $S$ by using his bargaining skills or any other means." Since the allocation corresponding to

\footnotetext{
${ }^{*}$ Takaaki Abe gratefully acknowledges the support of Grant-in-Aids for JSPS Research Fellow (DC1) No.16J00035. Shuige Liu gratefully acknowledges the support of Grant-in-Aids for Young Scientists (B) of JSPS No. 17K13707 and Grant for Special Research Project No. 2018K-016 of Waseda University.

${ }^{\dagger}$ Graduate School of Economics, Waseda University, 1-6-1 Nishi-Waseda, Shinjuku-Ku, 169-8050, Tokyo, Japan (takatomo3639@asagi.waseda.jp).

${ }^{\ddagger}$ School of Political Science and Economics, Waseda University, 1-6-1 Nishi-Waseda, Shinjuku-Ku, 169-8050, Tokyo, Japan (shuige_liu@aoni.waseda.jp).
} 
the grand coalition in a PMAS is always in the core, from the viewpoint of coalition formation, the existence of a PMAS guarantees a path leading to the grand coalition from any smaller one.

However, as Sprumont [9] himself pointed out, a critical drawback of PMAS is that it does not always exist. Worse, many market games, where coalition formation is a natural concern and the cores are non-empty, do not have any PMAS. A simple example is the $2 \times 2$ gloves game (Shapley and Shubik [7]). Further, Sprumont [9] showed that every assignment game with at least two sellers and two buyers also fails to have a PMAS.

This difficulty motivated us to modify the concept of PMAS by preserving its spirit of population monotonicity while relaxing its requirements to guarantee the existence in market games. In this paper, we introduce a concept called monotonic core allocation path (MCAP). A MCAP is a vector of allocations along an order on the set of players which satisfies (1) each allocation is in the core of the subgame corresponding to players at that step, and (2) the payoff for each player is monotonically non-decreasing in the process. Here, the coalition formation process can be thought as adding players one by one into the situation. In this context, the spirit that no player will ever be tempted to induce the formation of a smaller coalition boils down to the condition that no player would deviate at each step in the process. Further, the existence of a MCAP also guarantees a path to the formation of the grand coalition.

We use an example to show the basic idea. Consider the following two-sided matching situation with two buyers, 1,2 , and two sellers, $1^{\prime}, 2^{\prime}$ :

\begin{tabular}{l|l|l|}
\multicolumn{1}{l}{} & \multicolumn{1}{l}{$1^{\prime}$} & $2^{\prime}$ \\
\cline { 2 - 3 } 1 & 1 & 3 \\
\cline { 2 - 3 } 2 & 2 & 5 \\
\cline { 2 - 3 } & &
\end{tabular}

The number in each box is the payoff attainable from the exchange between the corresponding pair. Consider an order $\sigma=\left(1,1^{\prime}, 2,2^{\prime}\right)$. It can be seen that the following $\left(x^{\sigma_{k}}\right)_{k=1}^{4}$ is a MCAP on $\sigma$ :

$$
\begin{aligned}
x^{\sigma_{1}} & =(0), \\
x^{\sigma_{2}} & =(0,1), \\
x^{\sigma_{3}} & =(0,1,1), \\
x^{\sigma_{4}} & =(0,1,2,3) .
\end{aligned}
$$

where $\sigma_{k}$ is the $k$-th segment of $\sigma$, i.e, $\sigma_{1}=(1), \sigma_{2}=\left(1,1^{\prime}\right)$, etc. Each $x^{\sigma_{k}}(k=1, \ldots, 4)$ is a core allocation in the corresponding game. Player 1's payoff in the sequence is $0-0-0-0$, player $1^{\prime}$ is $1-1-1$, player 2 is $1-2$, and player $2^{\prime}$ is 3 ; all are non-decreasing.

However, the existence of such a sequence should not be taken for granted. For example, we cannot construct any MCAP on $\sigma$ if we start from a positive payoff of player 1 . The reason is that the occurrence of player 2 in $\sigma_{3}$, who can be seen as a substitute of player 1 , would draw player 1's payoff to 0 at $x^{\sigma_{3}}$.

This observation sheds doubt on the existence of a MCAP. Nevertheless, in this paper, we will show that MCAP exists for every assignment game. Our proof is based on a notion called tight graph introduced in Hammers et al. [3]. Given an assignment game and an extreme core allocation, a tight graph can be defined on the set of players where two players are connected if the sum of the payoffs they obtained in the allocation equals to the value attainable via their cooperation. An edge in the tight graph is thick with respect to an optimal matching if the two players are matched there and is thin if they are not. Based on a tight graph generated from an extreme core allocation where thick and thin edges are determined with respect to an optimal matching, we define an order on the set of players and show that the sequence obtained by expanding the extreme core allocation along the order is a MCAP. 
This result implies that for each extreme core allocation there is a MCAP converging to it. Though the reverse does not hold in general, we show that it holds for two special cases of assignment games, gloves games (Shapley and Shubik [7]) and Böhm-Bawerk games (see Shapley and Shubik [8]). It implies that not every core allocation can be approached by some MCAP. Therefore, the set of terminals of MCAPs is a refinement of the core.

The strong relationship with extreme core allocations may be taken as a drawback of the notion of MCAP since it implies that some players in such a path always attains his minimum possible payoff in the core. However, we claim that this is a deep insight on social welfare theory provided by MCAP, that is, a conflict between the stability of a coalition formation process and the fairness of the resulting outcomes. That a linear coalition formation process like MCAP which guarantees the stability by satisfying population monotonicity may only lead to extreme allocations suggests a general inconsistency between the social justice of procedure and outcome.

A further research in that direction is expected.

It is natural to wonder whether our results can be extended to general market games. Since our proofs are based on the tight graph which relies on the special structure of the assignment games, the possbility of generalization depends on whether we can construct a similar notion in market games, which is still an open problem. Nevertheless, we believe that our results can be preserved in general and anticipate further research in that direction.

In the literature of assignment games, orders on the set of players has long been employed in characterizing and searching extreme core allocations (Hammers et al. [3], Núñez and Rafels [4], Izquierdo et al. [5]). Our paper benefits from their results on tight graphs and zero-payoff players in extreme core allocations. However, orders on the set of players in those researches are basically auxiliaries in algorithms for generating a proper allocation, while in our paper, orders themselves are the focus. We interpret an order as a process of adding players into the situation, and investigate how to find the proper orders. Further, it can be shown that orders here for generating MCAPs are independent from benchmarks orders in their paper, for example, those which generates efficient max-payoff vectors (Izquierdo et al. [5]).

The rest of this paper is organized as follows. Section 2 presents preliminaries about cooperative game theory and assignment games; also, we introduce the notion of MCAP. Section 3 shows our main result, the existence of MCAP in every assignment games. Section 4 provides simpler ways to establish the existence of MCAP in gloves games and Böhm-Bawerk games.

\section{Preliminaries}

We begin with a short introduction on notions and results in cooperative game theory and assignment games. We also introduce the monotonic core allocation path.

A cooperative game with transferable utility (TU game) is a pair $(P, w)$ where $P$ is the finite set of players and $w: 2^{P} \rightarrow \mathbb{R}$, called the characteristic function, assigns each coalition $S \subseteq P$ a real number $w(S)$ and satisfies that $w(\emptyset)=0$. For each $S \subseteq P$, we use $w_{S}$ to denote the restriction of $w$ on $S$, that is, $w_{S}$ is the real-valued mapping from $2^{S}$ such that for each $T \subseteq S$, $w_{S}(T)=w(T)$.

An allocation of a TU game $(P, w)$ is a vector $x \in \mathbb{R}^{P}$. The core of $(P, w)$, denoted by $C(P, w)$, is defined as

$$
C(P, w)=\left\{x \in \mathbb{R}^{P} \mid \sum_{i \in P} x_{i}=w(P) \text { and } \sum_{i \in S} x_{i} \geq w(S) \text { for each } S \subseteq P\right\} .
$$

Each $x \in C(P, w)$ is called a core allocation. The core is the set of allocations which cannot be blocked by any coalition in $P$. The core of a TU game is not necessarily non-empty. Bondareva [1] and Shapley [6] show that a TU game has a non-empty core if and only if it satisfies a condition called balancedness. Allocation $x \in C(P, w)$ is called an extreme core allocation iff for 
each $y, z \in C(P, w), x=(1 / 2) y+(1 / 2) z$ implies that $y=z=x$. We use $\operatorname{ext}\{C(P, w)\}$ to denote the set of extreme core allocations of $(P, w)$. Since the core is a bounded convex polyhedra, $\operatorname{ext}\{C(P, w)\}$ is a finite set.

Assignment games, introduced by Shapley and Shubik [8], originate from two-sided matching situations. A two-sided matching situation is a tuple $\left(M, N,\left(a_{i j}\right)_{(i, j) \in M \times N}\right)$ where $M$ and $N$ are two finite disjoint sets and $a_{i j} \geq 0$ for each $(i, j) \in M \times N$. For each $S \subseteq M$ and $T \subseteq N$, a matching $\mu$ between $S$ and $T$ is a subset of $S \times T$ such that for each $(i, j),\left(i^{\prime}, j^{\prime}\right) \in \mu, i=i^{\prime}$ if and only if $j=j^{\prime}$. We use $\mathcal{M}(S, T)$ to denote the set of matchings between $S$ and $T$. Given a two-sided matching situation $\left(M, N,\left(a_{i j}\right)_{(i, j) \in M \times N}\right)$, we can induce a TU game $(M \cup N, w)$, called the assignment game, satisfying that for each $S \subseteq M$ and $T \subseteq N$,

$$
w(S \cup T)=\max \left\{\sum_{(i, j) \in \mu} a_{i j} \mid \mu \in \mathcal{M}(S, T)\right\} .
$$

A matching $\mu \in \mathcal{M}(S, T)$ is said to be optimal iff $\Sigma_{(i, j) \in \mu} a_{i j}=w(S \cup T)$. To differentiate payoffs of players in the two groups $M$ and $N$, we sometimes use $(u, v)$ instead of $x$ to denote an allocation in an assignment game, where $u \in \mathbb{R}^{M}$ and $v \in \mathbb{R}^{N}$.

Shapley and Shubik [8] show that the core of an assignment games is always non-empty. They also show the relationship between core allocations and optimal matchings, which is summarized in the following lemma. Here we follow the formulation of Hammers et al. [3].

Lemma 2.1 (Core allocations and optimal matchings). Consider a two-sided matching situation $\left(M, N,\left(a_{i j}\right)_{(i, j) \in M \times N}\right)$, the corresponding assignment game $(M \cup N, w)$, and an optimal matching $\mu \in \mathcal{M}(M, N)$. Then $x \in \mathbb{R}^{M \times N}$ is a core allocation if and only if the following four conditions are satisfied:

(i) $x_{i}+x_{j}=a_{i j}$ for all $(i, j) \in \mu$;

(ii) $x_{i}+x_{j} \geq a_{i j}$ for all $i \in M, j \in N$, and $(i, j) \notin \mu$;

(iii) $x_{k}=0$ for all unmatched players $k$;

(iv) $x_{k} \geq 0$ for all matched players $k$.

Consider the following question: given an assignment game $(M \cup N, w)$ and an order $\sigma=$ $\left(i_{1}, \ldots, i_{|M \cup N|}\right)$ on players, if we add players one by one into the situation along $\sigma$, is there a corresponding sequence of allocations satisfying that (1) each allocation is in the core of the corresponding subgame, and (2) the payoffs for each player are non-decreasing in such a sequence? To answer this question, we will introduce a concept called monotonic core allocation path.

An order on $M \cup N$ is a bijection $\sigma:\{1, \ldots,|M \cup N|\} \rightarrow M \cup N$. Here $|M \cup N|$ is the cardinality of $M \cup N$. As we did in the previous paragraph, sometimes we write an order $\sigma$ as $\left(i_{1}, \ldots, i_{|M \cup N|}\right)$ where $i_{1}=\sigma(1), i_{2}=\sigma(2)$, etc. For each $k=1, \ldots,|M \cup N|$, we use $\sigma_{k}$ to denote the restriction of $\sigma$ on $\{1, \ldots, k\}$, i.e., $\sigma_{k}=\left(i_{1}, \ldots, i_{k}\right)$; also, we use $R\left(\sigma_{k}\right)$ to denote the range of $\sigma_{k}$, i.e., $R\left(\sigma_{k}\right)=\left\{i_{1}, \ldots, i_{k}\right\}$. It is clear that $R\left(\sigma_{k}\right) \subseteq R\left(\sigma_{k+1}\right)$ for each $k=1, \ldots,|M \cup N|-1$. For each $\sigma_{k}$, we use $x^{\sigma_{k}}$ to denote a vector in $\mathbb{R}^{R\left(\sigma_{k}\right)}$, i.e., $x^{\sigma_{k}}=\left(x_{\sigma(1)}^{\sigma_{k}}, \ldots, x_{\sigma(k)}^{\sigma_{k}}\right)$.

Definition 2.1 (Monotonic core allocation path). Consider an assignment game $(M \cup N, w)$ and an order $\sigma=\left(i_{1}, \ldots, i_{|M \cup N|}\right)$ on $M \cup N$. A monotonic core allocation path (MCAP) over $\sigma$ is a sequence $\left(x^{\sigma_{k}}\right)_{k=1}^{|M \cup N|}$ satisfying the following two conditions:

M1. For each $k=1, \ldots,|M \cup N|, x^{\sigma_{k}}$ is a core allocation of the game $\left(R\left(\sigma_{k}\right), w_{R\left(\sigma_{k}\right)}\right)$;

M2. For each $k=1, \ldots,|M \cup N|-1$, and $i \in R\left(\sigma_{k}\right), x_{i}^{\sigma_{k}} \leq x_{i}^{\sigma_{k+1}}$.

M1 means that the $x^{\sigma_{k}}$ is a sequence of core allocations of the corresponding subgames of $(M \cup N, w)$. M2 means that each player's payoff is non-decreasing in the sequence. 


\section{Existence of a Monotonic Core Allocation Path}

In this section, we show our main theorem about the existence of MCAP.

Theorem 3.1 (Existence of a MCAP). Every assignment game has a MCAP.

Our proof is based on a notion called tight map introduced in Hammers et al. [3]. Consider an assignment game $(M \cup N, w)$ and an optimal matching $\mu \in \mathcal{M}(M, N)$. Given a core allocation $(u, v) \in \mathbb{R}^{M \cup N}$, we define a tight graph $G^{w}(u, v)=(V, E)$ where $V=M \cup N$ and $E=\left\{\{i, j\}: i \in M, j \in N\right.$, and $\left.u_{i}+v_{j}=a_{i j}\right\}$. In $G^{w}(u, v)$, all edges corresponding to $\mu$ are called thick edges and the other edges are called thin edges. Hammers et al. [3] show the following result.

Lemma 3.1 (Tight graphs and extreme core allocations). Let $(M \cup N, w)$ be an assignment game and $\mu$ be an optimal matching between $M$ and $N$. An allocation $(u, v)$ belongs to $\operatorname{ext}\{C(M \cup N, w)\}$ if and only if each component of $G^{w}(u, v)$ contains at least one player with payoff equal to zero.

Recall that a component of a graph is a maximal connected subgraph of the graph. ${ }^{1}$ We call a player who receives payoff 0 in $(u, v)$ a zero-payoff player $(Z P P)$ in $(u, v)$. A direct implication of Lemma 3.1 is that each $(u, v) \in \operatorname{ext}\{C(M \cup N, w)\}$ has at least one ZPP.

We have the following statement.

Lemma 3.2 (A ZPP-rooted tree on a component). Consider an allocation $(u, v) \in$ $\operatorname{ext}\{C(M \cup N, w)\}$ and the tight graph $G^{w}(u, v)=(V, E)$. Let $\left(V^{\prime}, E^{\prime}\right)$ be a component of $G^{w}(u, v)$ and $i^{*}$ a ZPP in $\left(V^{\prime}, E^{\prime}\right)$. Then we can construct a tree $\left(V^{\prime}, T\right)$ satisfying the following conditions:

T1. $\left(V^{\prime}, T\right)$ is a subgraph of $\left(V^{\prime}, E^{\prime}\right)$ contains every point in $V^{\prime}$;

T2. $\left(V^{\prime}, T\right)$ contains every thick edge in $\left(V^{\prime}, E^{\prime}\right)$;

T3. $i^{*}$ is the root of $\left(V^{\prime}, T\right)$.

Such a tree is called a ZPP-rooted tight tree on $\left(V^{\prime}, E^{\prime}\right)$.

Proof. This statement holds directly from Hammers et al. [3]'s result that each component contains a tight tree, i.e., a tree containing every thick edges with respect to an optimal matching $\mu$. Since a tree is a connected acyclic graph, we can specify $i^{*}$ to be its root.

Fix $(u, v) \in \operatorname{ext}\{C(M \cup N, w)\}$ and $G^{w}(u, v)=(V, E)$. Without loss of generality, we assume that $(V, E)$ is connected, i.e., $(V, E)$ is a component of itself. Let $i^{*}$ be a ZPP of $(u, v)$, and $(V, T)$ be a ZPP-rooted tight tree on $(V, E)$ with $i^{*}$ as its root. A tree-order associated to $(V, T)$ and $i^{*}$ is defined on $V$ as follows: for each $i, j \in V, i \leq j$ iff $i$ is on the path from $i^{*}$ to $j$. Since a rooted tree is an aborescence, $\leq$ is well defined. We use $<$ to denote the asymmetric part of $\leq$. For each $i \in V$, we define $\lceil i\rceil=\{j \in V \mid j \leq i\}$ and $\lfloor i\rfloor=\{j \in V \mid j \geq i\}$. An immediate successor (predecessor) of $i$ is a minimal element in $\lfloor i\rfloor \backslash\{i\}$ (a maximal element in $\lceil i\rceil \backslash\{i\}$ ). The set of immediate successors (predecessors) of $i$ is denoted by $\lfloor i\rfloor^{*}\left(\lceil i\rceil^{*}\right)$. For each $i \in V$, we define $\lfloor i\rfloor_{+}^{*}=\left\{j \in\lfloor i\rfloor^{*} \mid\{i, j\}\right.$ is a thick edge $\}$ and $\lfloor i\rfloor_{-}^{*}=\left\{j \in\lfloor i\rfloor^{*} \mid\{i, j\}\right.$ is a thin edge $\}$. It is clear that $\lfloor i\rfloor^{*}=\lfloor i\rfloor_{+}^{*} \cup\lfloor i\rfloor_{-}^{*}$.

We now define an order $\sigma$ on $V$ (i.e., $M \cup N$ ) as follows. First, let $\sigma(1)=i^{*}$. Suppose that $\sigma(k)$ is defined for some $k=1, \ldots,|M \cup N|-1 . \sigma(k+1)$ is defined as follows: when $\lfloor\sigma(k)\rfloor \neq \emptyset$, we let

$$
\sigma(k+1)=\left\{\begin{array}{lr}
\text { any } i \in\lfloor\sigma(k)\rfloor_{-}^{*} \\
\text { any } i \in\lfloor\sigma(k)\rfloor_{+}^{*}
\end{array} \quad \text { if }\lfloor\sigma(k)\rfloor_{-}^{*}=\emptyset \text { and }\lfloor\sigma(k)\rfloor_{+}^{*} \neq \emptyset .\right.
$$

If $\lfloor\sigma(k)\rfloor=\emptyset$, we define $\sigma(k+1)$ to be some $i \in V \backslash R\left(\sigma_{k}\right)$ satisfying the following conditions:

D1. $\lceil i\rceil^{*} \subseteq R\left(\sigma_{k}\right)$;

\footnotetext{
${ }^{1}$ For a detailed introduction of concepts in graph theory, see, for example, Diestel [2].
} 
D2. $\lceil i\rceil^{*}$ is a maximal element in the set $\left\{j \mid j \in R\left(\sigma_{k}\right)\right.$ and $\left.\lfloor j\rfloor^{*} \nsubseteq R\left(\sigma_{k}\right)\right\}$.

D2 requires that $\sigma(k+1)$ to be an immediate successor of a maximal branching point in $R\left(\sigma_{k}\right)$ whose offsprings are not all contained in $R\left(\sigma_{k}\right)$. In this manner, we define an order $\sigma$ on $V$. Order $\sigma$ can be regarded as a sequence of linear orders, that is, for each $i \in V$, there is a segment in $\sigma$ containing $i$ which is linearly ordered in $(V, T)$. It can be seen that the maximal linear segment is always ended with a leaf in $(V, T),{ }^{2}$ and each $i \in V$ has only one such leaf, called the leaf of $i$ in $(V, T)$ with respect to $\sigma$.

We use the following example to show how the definition works.

Example 3.1. Consider the following two-sided matching situation:

\begin{tabular}{|c|c|c|c|}
\hline & $1^{\prime}$ & $2^{\prime}$ & $3^{\prime}$ \\
\hline 1 & 6 & 2 & 1 \\
\hline 2 & 9 & 8 & 3 \\
\hline 3 & 7 & 5 & 2 \\
\hline
\end{tabular}

The allocation $(u, v)=(0,3,1 ; 6,5,1)$ is in the core and $\mu=\left\{\left(1,1^{\prime}\right),\left(2,2^{\prime}\right),\left(3,3^{\prime}\right)\right\}$ is an optimal matching. The tight graph $G^{w}(u, v)$ is depicted on the left-hand side of Figure 3.1. On the right-hand side we give two ZPP-rooted trees $(V, T)$ and $\left(V, T^{\prime}\right)$ with player 1 as the root.
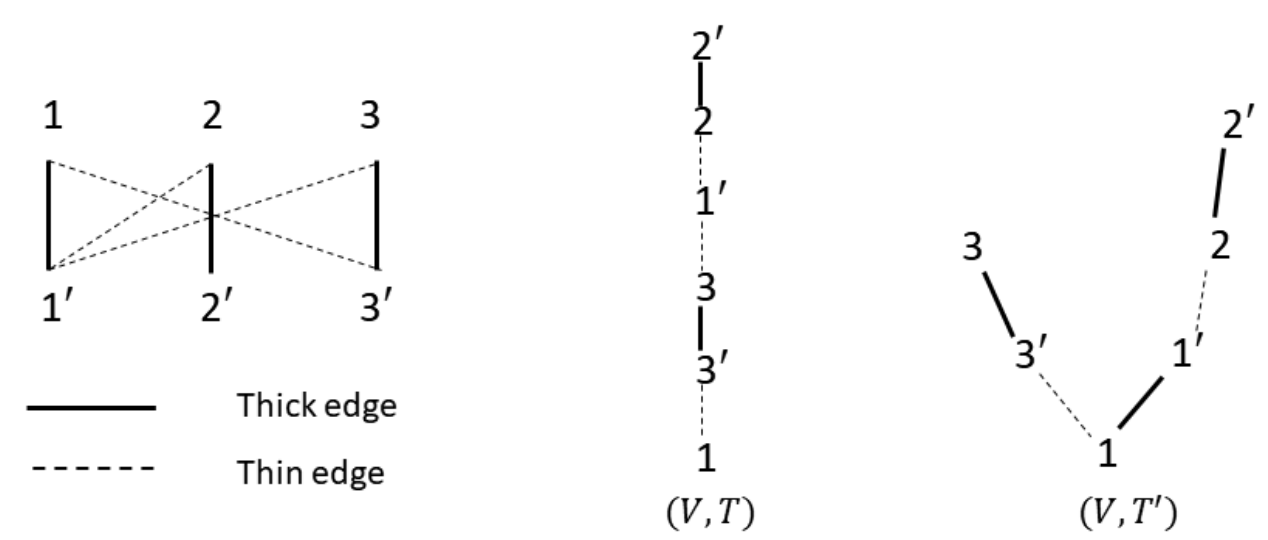

Figure 3.1. $G^{w}(u, v)$ and two ZPP-rooted trees

Both trees lead to the order $\sigma=\left(1,3^{\prime}, 3,1^{\prime}, 2,2^{\prime}\right)$. Note that though $\left(V, T^{\prime}\right)$ contains two branches, by (3.1) we first have to exhaust $1-3^{\prime}-3$ since $\left\{1,3^{\prime}\right\}$ is thin and $\left\{1,1^{\prime}\right\}$ is thick. Nevertheless, in general, a tree may allow several orders. For example, suppose there are three branches from $i^{*}$ where two are thin and one is thick. By (3.1) we can exhaust either thin edge first.

Consider $\sigma$ and $\left(V, T^{\prime}\right)$. The order $\sigma$ is composed of two linear orders in $\left(V, T^{\prime}\right)$, that is, $\left(1,3^{\prime}, 3\right)$ and $\left(1^{\prime}, 2,2^{\prime}\right)$. For players in the first group, their leaf in $\left(V, T^{\prime}\right)$ with respect to $\sigma$ is 3 ; for the second group it is $2^{\prime}$.

We have the following statement.

Lemma 3.3 (Extreme core allocations, ZPP-rooted tight tree orders, and MCAP) Consider $x=(u, v) \in \operatorname{ext}\{C(M \cup N, w)\}$, an optimal matching $\mu$, and an order $\sigma$ generated from a ZPP-rooted tight tree on $G^{w}(u, v)$. For each $k=1, \ldots,|M \cup N|$, let $x^{\sigma_{k}}=\left(x_{i}\right)_{i \in R\left(\sigma_{k}\right)}$. Then $\left(x^{\sigma_{k}}\right)_{k=1}^{|M \cup N|}$ is a MCAP.

To prove Lemma 3.3, we need to show that for each $k=1, \ldots,|M \cup N|$, there is an optimal matching in the game $\left(R\left(\sigma_{k}\right), w_{R\left(\sigma_{k}\right)}\right)$ which "supports" $x^{\sigma_{k}}$. Consider Example 3.1 again. Allocation $x^{\sigma_{2}}$ is supported by the matching $\left\{\left(1,3^{\prime}\right)\right\}, x^{\sigma_{3}}$ by $\left\{(1),\left(3,3^{\prime}\right)\right\}$ (i.e., player 1 is matched

\footnotetext{
${ }^{2}$ Recall that a leaf in a tree is a vertex which has no successor.
} 
with nobody and player 3 is matched with $\left.3^{\prime}\right), x^{\sigma_{4}}$ by $\left\{\left(1,1^{\prime}\right),\left(3,3^{\prime}\right)\right\}, x^{\sigma_{5}}$ by $\left\{(1),\left(3,3^{\prime}\right),\left(1^{\prime}, 2\right)\right\}$, and $x^{\sigma_{6}}$ by $\left\{\left(1,1^{\prime}\right),\left(2,2^{\prime}\right),\left(3,3^{\prime}\right)\right\}$. A player may change partners in the process and, sometimes even though her partner in the "final" matching appears, they may not always be matched together. In our proof, we will provide the general law which determines the optimal matching supporting $x^{\sigma_{k}}$ at each step $k$.

Proof of Lemma 3.3. Let $(V, T)$ be a ZPP-rooted tight tree on $G^{w}(u, v)$ with ZPP $i^{*}$ as its root from which $\sigma$ is generated. For each $i \in V$, let $T(i)$ be the set of all edges connecting $i$ and $i^{*}, T(i)_{+}$be the set of all thick edges in $T(i)$, and $T(i)_{-}$be the set of all thin edges in $T(i)$. It is clear that edges in $T(i)$ can also be ordered by the tree order from $(V, T)$. For each $\ell=1, \ldots,|T(i)|$, we use $e_{\ell}$ to denote the $\ell$-th edge in $T(i)$.

Based on $\sigma$, we define an order $\prec_{\sigma}$ on the set of leafs in $(V, T)$ as follows: for leafs $j, j^{\prime} \in V$, $j \prec_{\sigma} j^{\prime}$ iff $\sigma^{-1}(j)<\sigma^{-1}\left(j^{\prime}\right)$.

Now we show that for each $k=1, \ldots,|M \cup N|$, there is an optimal matching in the game $\left(R\left(\sigma_{k}\right), w_{R\left(\sigma_{k}\right)}\right)$ which supports $x^{\sigma_{k}}$. It is straightforward that $\left\{\left(i^{*}\right)\right\}$ supports $x^{\sigma_{1}}$. Let $k \in$ $\{1, \ldots,|M \cup N|-1\}, i=\sigma(k+1)$, and $j$ be the leaf of $i$ in $(V, T)$ with respect to $\sigma$. Consider the following two sets of edges:

$$
\begin{gathered}
A(i):=\left(\cup_{j^{\prime} \prec_{\sigma} j} T_{+}\left(j^{\prime}\right)\right) \backslash T_{+}(i) . \\
B(i):=\left\{\begin{array}{cc}
\left\{e_{1}, e_{3}, \ldots, e_{|V(i)|-1}\right\} & \text { if }|T(i)| \text { is odd, } \\
\left\{e_{2}, e_{4}, \ldots, e_{|V(i)|-1}\right\} & \text { if }|T(i)| \text { is even. }
\end{array}\right.
\end{gathered}
$$

Let $S(i)=\left\{\left(i^{\prime}\right) \mid i^{\prime} \in R(\sigma(k+1))\right.$ and $i^{\prime}$ does not belong to any edge in $\left.A(i) \cup B(i)\right\}$. It can be seen that $A(i) \cup B(i) \cup S(i)$ is an optimal matching in $\left(R\left(\sigma_{k+1}\right), w_{R\left(\sigma_{k+1}\right)}\right)$ which supports $x^{\sigma_{k+1}}$. To see this, we only need to show that for each $i^{\prime} \in R\left(\sigma_{k+1}\right), i^{\prime} \in S(i)$ only if it is a ZPP in $x^{\sigma_{k+1}}$. Suppose $i^{\prime}$ is not a ZPP. We discuss the following two cases:

Case 1. Player $i^{\prime}$ does not belong to any edge in $T(i)$. Since $i^{\prime}$ is not a ZPP, by Lemma 2.1, $i^{\prime}$ is matched to some player $i^{\prime \prime}$ in $\mu$. Recall the definition of a ZPP-rooted tight tree which requires that each thick edge should be contained in $(V, T)$. If $i^{\prime}$ does not belong to any edge in $T(i)$, neither does $i^{\prime \prime}$. Therefore, $\left\{i^{\prime}, i^{\prime \prime}\right\} \in A(i)$ and that is the only edge associated with $i^{\prime}$ in $A(i)$.

Case 2. Player $i^{\prime}$ belongs to some edge in $T(i)$. By (3.3) the only player which may not be matched (when $T(i)$ is even) is $i^{*}$. Since $i^{*}$ is a ZPP, $i^{\prime} \neq i^{*}$, and consequently $i$ is matched with some player in $B(i)$ (not necessarily his partner in $\mu$ ).

Here we have shown that $A(i) \cup B(i) \cup S(i)$ is an optimal matching in $\left(R\left(\sigma_{k+1}\right), w_{R\left(\sigma_{k+1}\right)}\right)$ which supports $x^{\sigma_{k}}$. Therefore, $x^{\sigma_{k}}$ is a core allocation in $\left(R\left(\sigma_{k+1}\right), w_{R\left(\sigma_{k+1}\right)}\right)$.

Proof of Theorem 3.1. Consider $x=(u, v) \in \operatorname{ext}\{C(M \cup N, w)\}$, an optimal matching $\mu$, a ZPP-rooted tight tree $G^{w}(u, v)$. Let $\left(\left(V^{1}, E^{1}\right), \ldots,\left(V^{L}, E^{L}\right)\right)$ be a sequence of components in $G^{w}(u, v)$. By Lemma 3.1, for each $\ell=1, \ldots, L$, there is some ZPP $i^{\ell} \in V^{\ell}$. Then we can define a ZPP-rooted tight tree $\left(V^{\ell}, T^{\ell}\right)$ on $\left(V^{\ell}, E^{\ell}\right)$ with $i^{\ell}$ as its root for each $\ell$, based on which an order $\sigma^{\ell}$ on $V^{\ell}$ can be defined. Let $\sigma=\left(\sigma^{1}, \ldots, \sigma^{L}\right)$. Since each component is independent from others, it follows from Lemma 3.3 that $\left(x^{\sigma_{k}}\right)_{k=1}^{|M \cup N|}$ is a MCAP.

From the proof of Lemma 3.3 we have the following corollary.

Corollary 3.1 (Extreme core allocations and MCAP) For each $x=(u, v) \in \operatorname{ext}\{C(M \cup$ $N, w)\}$ there is an order $\sigma$ on $M \cup N$ such that $\left(x^{\sigma_{k}}\right)_{k=1}^{|M \cup N|}$ is a MCAP.

We can define a refinement of the core by MCAP as follows. An allocation $x \in C(M \cup N, w)$ is called a MCAP core allocation iff there is some MCAP $\left(x^{\sigma_{k}}\right)_{k=1}^{|M \cup N|}$ such that $x=x^{\sigma|M \cup N|}$. Corollary 3.1 implies that each extreme core allocation is a MCAP core allocation. Its converse is not true. Consider the example in Section 1. The allocation $x$ which assigns 0.5 to player 
1 and $1^{\prime}$ respectively, 2 to player 2 , and 3 to player $2^{\prime}$ is in the core but not an extreme core allocation according to Lemma 3.1. Consider $\sigma=\left(2,2^{\prime}, 1,1^{\prime}\right)$ and $\left(x^{\sigma_{k}}\right)_{k=1}^{4}$ as follows:

$$
\begin{aligned}
x^{\sigma_{1}} & =(0), \\
x^{\sigma_{2}} & =(2,3), \\
x^{\sigma_{3}} & =(2,3,0), \\
x^{\sigma_{4}} & =(2,3,0.5,0.5) .
\end{aligned}
$$

It can be seen that $\left(x^{\sigma_{k}}\right)_{k=1}^{4}$ is a MCAP. Here we have shown that $x\left(=x^{\sigma_{4}}\right)$ is a MCAP core allocation but not an extreme core allocation.

It is then natural to wonder whether every core allocation can be approached by some MCAP. In Section 4 we will show that this conjecture is not true. There, we will characterize MCAP allocations in a subclass of assignment games.

\section{MCAP in Gloves Games and Böhm-Bawerk Games}

In Section 3, we have established the existence of MCAP in every assignment game. This section shows that the existence result can be shown in simpler ways for two special cases of assignment games: gloves games and Böhm-Bawerk games. Especially, in Subsection 4.1 we will show that some core allocation cannot be approached by any MCAP.

\subsection{MCAP in gloves games}

Gloves games, introduced in Shapley and Shubik [7], are the simplest assignment games. A gloves game is a pair $(L \cup R, w)$ where $L=\left\{l_{1}, \ldots, l_{|L|}\right\}$ and $R=\left\{r_{1}, \ldots, r_{|R|}\right\}$ are interpreted as the sets of right-hand and left-hand gloves respectively. Since each pair of gloves has the same value which can be normalized to be 1 and a single glove is worthless, the value of each coalition in $L \cup R$ is the number of pairs it contains. Formally, for each $S \subseteq L \cup R, w(S)=\min \{|S \cap L|,|S \cap R|\}$. Without loss of generality, we assume that $|L| \leq|R|$. Consider the following allocation $x \in \mathbb{R}^{L \cup R}$ : for each $i \in L \cup R$,

$$
x_{i}=\left\{\begin{array}{l}
1 \text { if } i \in L, \\
0 \text { if } i \in R .
\end{array}\right.
$$

It is clear that $x \in C(L \cup R, w)$. Consider an order $\sigma=\left(r_{1}, l_{1}, r_{2}, l_{2}, \ldots, r_{|L|}, l_{|L|}, r_{|L+1|}, \ldots, r_{|R|}\right)$, that is, starting from a player in $R$, players in $R$ and $L$ occur alternatively until the set $L$ is exhausted, and then only players in $R$ until it is also exhausted. It can be seen that $\left(x^{\sigma_{k}}\right)_{k=1}^{|L \cup R|}$ is a MCAP.

Actually, $\sigma$ is an order generated from a special ZPP-tight tree. Under the core allocation $x$ defined in (4.1), every $(l, r) \in L \times R$ is an edge in the tight tree $G^{w}(x)$. Given an optimal matching $\mu=\left\{\left(l_{k}, r_{k}\right)\right\}_{k=1}^{|L|} \cup\left\{\left(r_{k}\right)\right\}_{k=|L|+1}^{|R|}$, each $(l, r) \in L \times R$ is a thick edge if and only if $l=l_{k}$ and $r=r_{k}$ for some $k=1, \ldots,|L|$. Consider the tree $(L \cup R, T)$ with $l_{1}$ as its root such that $T=\left\{\left(l_{k}, r_{k}\right)\right\}_{k=1}^{|L|} \cup\left\{\left(r_{k}, l_{k+1}\right)\right\}_{k=1}^{|L|-1} \cup\left\{\left(r_{k}\right)\right\}_{k=|L|+1}^{|R|}$. It can be seen that $(L \cup R, T)$ is a ZPP-rooted tight tree and $\sigma$ is an order generated from it.

We can see from gloves games that some core allocation cannot be approached by any MCAP. For example, consider the gloves game with two left-hand gloves and two right-hand ones. The allocation $y=(1 / 2,1 / 2,1 / 2,1 / 2)$ is in the core. However, there is no MCAP $\left(x^{\sigma_{k}}\right)_{k=1}^{4}$ satisfying that $x^{\sigma_{4}}=y$. Indeed, for each order $\sigma, \sigma_{3}$ contains two gloves for one side and a single glove for the other side. Hence, the only core allocation for $\left(R\left(\sigma_{3}\right), w_{R\left(\sigma_{3}\right)}\right)$ gives the single glove holder payoff 1 and 0 to the others, which implies that the payoffs for the single glove holder strictly decreases from $x^{\sigma_{3}}$ to $x^{\sigma_{4}}$. In this sense, the set of terminals of MCAPs is a refinement of the core. 
By generalizing the above argument, we have the following statement which characterizes MCAP core allocations in gloves games.

Proposition 4.1 (Characterizing MCAP core allocations in gloves games) Let $(L \cup$ $R, w)$ be a gloves game with $\max \{|L|,|R|\} \geq 2$. An allocation $x$ is a MCAP core allocation if and only if it is an extreme core allocation.

Though there is a straightforward proof, we omit it here since this statement is a direct implication of Proposition 4.2.

\subsection{MCAP in Böhm-Bawerk Games}

Böhm-Bawerk games, which arise from Böhm-Bawerk's study on horse markets (see Shapley and Shubik [8]), generalize the gloves games and form a special case of assignment games. A BöhmBawerk horse market (BB market) is a tuple $\left(M, N,\left\{h_{i}\right\}_{i \in M},\left\{c_{j}\right\}_{j \in N}\right)$, where $M=\{1, \ldots, m\}$ is the set of buyers, $N=\left\{1^{\prime}, \ldots, n^{\prime}\right\}$ the set of sellers, $h_{i}$ is the evaluation of buyer $i$ about a horse and $c_{j}$ is that of seller $j$ about his horse. Without loss of generality, we assume that $h_{i} \geq h_{i+1}$ for each $i=1, \ldots, m$ and $c_{j} \leq c_{j+1}$ for each $j=1^{\prime}, \ldots, n^{\prime}$. From a BB market $\left(M, N,\left\{h_{i}\right\}_{i \in M},\left\{c_{j}\right\}_{j \in N}\right)$, we define a TU game $(M \cup N, w)$, called the Böhm-Bawerk game (BB game), satisfying that for each $S \subseteq M$ and $T \subseteq N$,

$$
w(S \cup T)=\max \left\{\Sigma_{(i, j) \in \mu}\left(h_{i}-c_{j}\right): \mu \in \mathcal{M}(S, T)\right\} .
$$

We will give a simple and intuitive order on $M \cup N$ and show that there is a MCAP on it. Let $q=\max \left\{i \mid h_{i} \geq c_{i^{\prime}}\right\}$. We discuss the following two cases:

Case 1. $\min \left\{h_{q}, c_{(q+1)^{\prime}}\right\}=h_{q}$. Then $q$ is called the marginal buyer. Define an allocation $x$ as follows: for each $i \in M \cap N$,

$$
x_{i}=\left\{\begin{array}{c}
\max \left\{h_{i}-h_{q}, 0\right\} \text { if } i \in M \\
\max \left\{h_{q}-c_{i}, 0\right\} \text { if } i \in N .
\end{array}\right.
$$

It is clear that $x \in \operatorname{ext}\{C(M \cup N, w)\}$ (and buyer $q$ gets zero-payoff), which is supported by the optimal matching $\mu=\left\{\left(i, i^{\prime}\right) \mid i=1, \ldots, q\right\} \cup\{(i) \mid i>q\} \cup\left\{\left(i^{\prime}\right) \mid i>q\right\}$. Consider an order $\sigma=\left(q,(q-1)^{\prime},(q-1), \ldots, 1^{\prime}, 1, q^{\prime}, \ldots\right)$ where after $q^{\prime}$ it can be any order on the remaining players. For each $k=1, \ldots,|M \cup N|$, let $x^{\sigma_{k}}$ be the restriction of $x$ on $R\left(\sigma_{k}\right)$. We can see that $\left(x^{\sigma_{k}}\right)_{k=1}^{|M \cup N|}$ is a MCAP. Indeed, for each $k, x^{\sigma_{k}}$ is supported by the following matching:

(i) $\left\{(q),\left((q-1)^{\prime},(q-1)\right), \ldots,\left(\left(q-\frac{k-1}{2}\right)^{\prime},\left(q-\frac{k-1}{2}\right)\right)\right\}$ if $k \leq 2 q-1$ and $k$ is odd;

(ii) $\left\{\left((q-1)^{\prime},(q-1)\right), \ldots,\left(\left(q-\frac{k-2}{2}\right)^{\prime},\left(q-\frac{k-2}{2}\right)\right),\left(q,\left(q-\frac{k}{2}\right)\right)\right\}$ if $k \leq 2 q-1$ and $k$ is even;

(iii) $\left\{\left(q^{\prime}, q\right), \ldots\left(1,1^{\prime}\right), \ldots\right\}$ if $k \geq 2 q$, where after $\left(1,1^{\prime}\right)$ is the sequence of single players until the $k$-th one in $\sigma$.

The optimal matchings supporting each $x^{\sigma_{k}}$ is illustrated in Figure 4.1. 


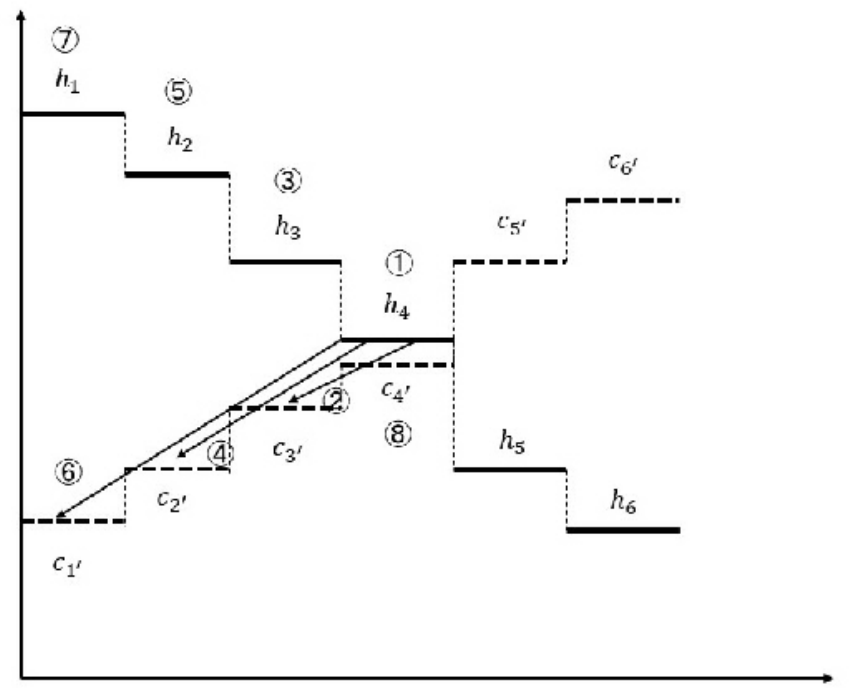

Figure 4.1. The matchings supporting a MCAP in a BB game

Here $q=4$. The numbers in the circles are the order of matchings in $\sigma$. We start from ZPP buyer 4 . At first buyer 4 matches with seller $3^{\prime}$, then the matching partner of $3^{\prime}$, i.e, buyer 3 , comes and matches with $3^{\prime}$. Then buyer 4 matched with seller $2^{\prime}$, and similarly the matching partner of $2^{\prime}$, i.e., buyer 2 , comes and matches with $2^{\prime}$, etc. Finally all players smaller than 4 are matched. Then comes seller $4^{\prime}$ who matches with buyer 4 . After that, those unmatched players come with any order.

Case 2. $\min \left\{h_{q}, c_{(q+1)^{\prime}}\right\}=c_{(q+1)^{\prime}}$. This is similar to Case 1. Now $(q+1)^{\prime}$ is the marginal seller. Define an allocation $x$ as follows: for each $i \in M \cap N$,

$$
x_{i}=\left\{\begin{array}{c}
\max \left\{h_{i}-c_{(q+1)^{\prime}}, 0\right\} \text { if } i \in M, \\
\max \left\{c_{(q+1)^{\prime}}-c_{i}, 0\right\} \text { if } i \in N .
\end{array}\right.
$$

It is clear that $x$ is the unique core allocation (and seller $(q+1)^{\prime}$ gets zero-payoff) which is supported by the optimal matching $\mu=\left\{\left(i, i^{\prime}\right) \mid i=1, \ldots, q\right\} \cup\{(i) \mid i>q\} \cup\left\{\left(i^{\prime}\right) \mid i>q\right\}$. Consider an order $\sigma=\left((q+1)^{\prime}, q, q^{\prime}, \ldots, 1,1^{\prime}, \ldots\right)$ where after $1^{\prime}$ it can be any order on the remaining players. For each $k=1, \ldots,|M \cup N|$, let $x^{\sigma_{k}}$ be the restriction of $x$ on $R\left(\sigma_{k}\right)$. We can see that $\left(x^{\sigma_{k}}\right)_{k=1}^{|M \cup N|}$ is a MCAP. Indeed, for each $k, x^{\sigma_{k}}$ is supported by

(i) $\left\{\left((q+1)^{\prime}\right),\left(q^{\prime}, q\right), \ldots,\left(\left(q-\frac{k-3}{2}\right)^{\prime},\left(q-\frac{k-3}{2}\right)\right)\right\}$ if $k \leq 2 q+1$ and $k$ is odd ${ }^{3}$

(ii) $\left\{\left(q^{\prime}, q\right), \ldots,\left(\left(q-\frac{k-4}{2}\right)^{\prime},\left(q-\frac{k-4}{2}\right)\right),\left((q+1)^{\prime},\left(q-\frac{k-2}{2}\right)\right)\right\}$ if $k \leq 2 q+1$ and $k$ is even;

(iii) $\left\{\left(q^{\prime}, q\right), \ldots\left(1,1^{\prime}\right), \ldots\right\}$ if $k \geq 2 q+2$, where after $\left(1,1^{\prime}\right)$ is the sequence of single players until the $k$-th one in $\sigma$.

This is an analog of Case 1: as illustrated in Figure 4.1, the marginal player, which is a ZPP, is matched to each seller (buyer) before him, then gives his position to the seller's (buyer's) partner, and moves on to the next seller (buyer). The intuition behind this construction is exhibited in our proof of Lemma 3.3 (and therefore Theorem 3.1).

The following statement characterizes MCAP core allocations in BB games.

Proposition 4.2 (Characterizing MCAP core allocations in BB games) Let $(M \cup N, w)$ be a BB game with $\max \{|M|,|N|\} \geq 2$. An allocation $x$ is a MCAP core allocation if and only if it is an extreme core allocation.

\footnotetext{
${ }^{3}$ When $k=1$ we manipulate that the matching is $\left\{\left((q+1)^{\prime}\right)\right\}$.
} 
As Proposition 4.1, the if part of Proposition 4.2 follows from Corollary 3.1. To show the only-if part, we have to make it clear what is an extreme core allocation in a BB game.

Consider a BB game $(M \cup N, w)$ with $\max \{|M|,|N|\} \geq 2$. Let $q=\max \left\{i \mid h_{i} \geq c_{i^{\prime}}\right\}$. We define marginal players in the following four cases:

Case 1. When $\min \left\{h_{q}, c_{(q+1)^{\prime}}\right\}=h_{q}$ and $\max \left\{c_{q^{\prime}}, h_{q+1}\right\}=c_{q^{\prime}}$, we call buyer $q$ and seller $q^{\prime}$ the marginal players;

Case 2. When $\min \left\{h_{q}, c_{(q+1)^{\prime}}\right\}=c_{(q+1)^{\prime}}$ and $\max \left\{c_{q^{\prime}}, h_{q+1}\right\}=c_{q^{\prime}}$, we call sellers $q^{\prime}$ and $(q+1)^{\prime}$ the marginal players;

Case 3. When $\min \left\{h_{q}, c_{(q+1)^{\prime}}\right\}=h_{q}$ and $\max \left\{c_{q^{\prime}}, h_{q+1}\right\}=h_{q+1}$, we call buyers $q$ and $q+1$ the marginal players;

Case 4. When $\min \left\{h_{q}, c_{(q+1)^{\prime}}\right\}=c_{(q+1)^{\prime}}$ and $\max \left\{c_{q^{\prime}}, h_{q+1}\right\}=h_{q+1}$, we call buyer $q+1$ and seller $(q+1)^{\prime}$ the marginal players.

When buyer $q+1$ or seller $(q+1)^{\prime}$ does not exist, we manipulate $\max \left\{c_{q^{\prime}}, h_{q+1}\right\}=c_{q^{\prime}}$ or $\min \left\{h_{q}, c_{(q+1)^{\prime}}\right\}=h_{q}$, respectively. Given a BB game $(M \cup N, w)$ and the original BB horse market $\left(M, N,\left\{h_{i}\right\}_{i \in M},\left\{c_{j}\right\}_{j \in N}\right)$, each stable matching is supported by an equilibrium market price $p \in \mathbb{R}$ in the market, and the scale of $p$ is determined by the two marginal players.

We have the following statement.

Lemma 4.1 (Core allocations in BB games) Let $(M \cup N, w)$ be a BB game. An allocation $x \in C(M \cup N, w)$ is an extreme core allocation if and only at least one marginal player gets zero payoff.

Proof. Let $q=\max \left\{i \mid h_{i} \geq c_{i^{\prime}}\right\}$. Then buyers $1, \ldots, q$ and sellers $1^{\prime}, \ldots, q^{\prime}$ are matched players. We only show it for Cases 1 and 4; Cases 2 and 3 can be proved in a similar manner.

(If) For Case 1, it can be seen that in the tight graph $G^{w}(x)$, all matched players are connected, and consequently belong to the same component $(V, E)$. Since each marginal player in Case 1 is connected, that a marginal player gets zero payoff implies that some player in $(V, E)$ gets zero payoff. Further, since every unmatched player gets zero payoff in $x$, it follows from Lemma 3.1 that $x$ is an extreme core allocation.

For Case 4, without loss of generality, suppose that $x_{q+1}=0$, i.e., the buyer $q+1$ (a marginal player) gets zero payoff. As in Case 1, still all matched players are connected in the tight graph $G^{w}(x)$; further, now buyer $q+1$ is also connected to those matched players since $x_{q+1}+x_{q^{\prime}}=h_{q+1}-c_{q^{\prime}}$. Therefore, $x_{q+1}=0$ means that in the component containing all matched players, there is a player gets zero payoff. Since every unmatched player gets zero payoff in $x$, it follows from Lemma 3.1 that $x$ is an extreme core allocation.

(Only-if) For Case 1, suppose that each marginal player, i.e., buyer $q$ and seller $q^{\prime}$, gets a positive payoff in $x$. It can be seen that all matched players form a component in $G^{w}(x)$. However, since $x_{q}>0$ and $x_{q^{\prime}}>0$, it follows that every macthed player gets a positive payoff. By Lemma 3.1, it follows that $x \notin \operatorname{ext}\{C(M \cup N, w)\}$. For Case 4, the statement holds since at least one maginal player is unmatched.

Proof of Proposition 4.2. We show the only-if part. By Lemma 4.1, it is equivalent to show that for in each MCAP core allocation, at least one marginal player gets zero payoff. Let $(M \cup N, w)$ be a BB game with $\max \{|M|,|N|\} \geq 2$ and $\mu$ be an optimal matching. We start from the case that each player is matched. It follows that $|M|=|N|=m(\geq 2)$ and each buyer $i$ is matched to seller $i^{\prime}$ in $\mu$. Here, what we have to show is that buyer $m$ or seller $m^{\prime}$ gets zero payoff in each MCAP core allocation.

Consider an order $\sigma$ and a $\operatorname{MCAP}\left(x^{\sigma_{k}}\right)_{k=1}^{|M \cup N|}$. It is clear that the price supporting the matchings does not change at each $k$ whenever $R\left(\sigma_{k}\right) \cap M \neq \emptyset$ and $R\left(\sigma_{k}\right) \cap N \neq \emptyset$, otherwise some player's payoffs would decrease, which is a contradiction. Consider the two marginal players, buyer $m$ and seller $m^{\prime}$. Without loss of generality, we assume that the $m$ appears before $m^{\prime}$ in $\sigma$. Now we show that $x_{m}^{\sigma_{k}}=0$ for each $k$ with $m \in R\left(\sigma_{k}\right)$. 
Let $k^{*}=\min \left\{k \mid m \in R\left(\sigma_{k}\right)\right.$ and $\left.R\left(\sigma_{k}\right) \cap N \neq \emptyset\right\}$. It is clear that $k^{*} \geq 2$. What we have to show is that $x_{m}^{\sigma_{k^{*}}}=0$. Since, as we have shown, the price supporting the matchings does not change through the MCAP, $x_{m}^{\sigma_{k^{*}}}=0$ implies that $x_{m}^{\sigma_{k}}=0$ for each $k$ with $m \in R\left(\sigma_{k}\right)$. We discuss the following two cases:

Case 1. $R\left(\sigma_{k^{*}-1}\right) \cap N=\emptyset$. Here are two subcases:

Subcase 1. $R\left(\sigma_{k^{*}-1}\right) \backslash\{m\}=\emptyset$, i.e., there is no other buyer in $R\left(\sigma_{k^{*}-1}\right) .{ }^{4}$ Suppose that $x_{m}^{\sigma_{k^{*}}}>0$. Let $k^{* *}=\min \left\{k \mid\left(R\left(\sigma_{k}\right) \cap M\right) \backslash\{m\} \neq \emptyset\right\}$ and $\{i\}=R\left(\sigma_{k^{* *}}\right) \backslash R\left(\sigma_{k^{* *}-1}\right)$, i.e., $i$ is the first buyer who occurs after $m$ in $\sigma$. Since the price is the same at $k^{*}$ and $k^{* *}, x_{m}^{\sigma} \sigma^{* *}=x_{m}^{\sigma_{k^{*}}}>0$, that is, $m$ is matched to some $j^{\prime} \in N$ at $k^{* *}$. Since $m$ is a marginal buyer, $h_{i} \geq h_{m}$, it follows that $i$ and $j^{\prime}$ can deviate from the matching because the cooperation between them can acheive payoffs $x_{m}^{\sigma_{k^{* *}}} / 2$ for $i$ and $x_{j^{\prime}}^{\sigma_{k^{* *}}}+\left(x_{m}^{\sigma_{k^{* *}}} / 2\right)$ for $j^{\prime}$, which strictly improve their original payoffs 0 and $x_{j^{\prime}}^{\sigma_{k^{* *}}}$. This is a contradiction. Hence $x_{m}^{\sigma_{k^{*}}}=0$.

Subcase 2. $R\left(\sigma_{k^{*}-1}\right) \backslash\{m\} \neq \emptyset$, i.e., there is some other buyer $i \in M$ in $R\left(\sigma_{k^{*}-1}\right)$. Let $R\left(\sigma_{k^{*}}\right) \cap N=\left\{j^{\prime}\right\}$, i.e., $j^{\prime}$ is the first seller entering the situation by $\sigma$. We also suppose that $x_{m}^{\sigma_{k^{*}}}>0$, i.e., $m$ is matched to $j^{\prime}$. Since $m$ is a marginal buyer, $h_{i} \geq h_{m}$, by a similar argument as in Subcase 1, $i$ and $j^{\prime}$ can deviate from the matching, a contradiction. Hence $x_{m}^{\sigma_{k^{*}}}=0$.

Case 2. $m \notin R\left(\sigma_{k^{*}-1}\right)$. Here still we have two subcases. Subcase 1 is that $R\left(\sigma_{k^{*}-1}\right) \cap M=\emptyset$. By a similar argument as in Sebcase 1 of Case 1, it can be seen that $x_{m}^{\sigma_{k^{*}}}=0$. Here we only discuss Subcase 2: $R\left(\sigma_{k^{*}-1}\right) \cap M \neq \emptyset$., i.e., there is some seller(s) and buyer(s) other than $m$ in $R\left(\sigma_{k^{*}-1}\right)$. Suppose that $x_{m}^{\sigma_{k^{*}}}>0$, i.e., $m$ is matched to some $j^{\prime} \in N$ at $k^{*}$. It is impossible that some other buyer $i$ is unmatched at $k^{*}$, otherwise $i$ can deviate together with the seller matched to $m$. On the other hand, since $m^{\prime} \notin R\left(\sigma_{k^{*}}\right)$ and $|M|=|N|$, there must still be some buyer who are not contained in $R\left(\sigma_{k^{*}}\right)$. It is clear that the first buyer appearing after $m$ can deviate toether with $m$ 's partner, which is a contradiction. Hence $x_{m}^{\sigma_{k^{*}}}=0$.

General cases can be shown in a similar manner. It can be seen that non-marginal unmatched players do not affect the situation. Hence in each MCAP core allocation at least one marginal player gets zero payoff. Then the statement holds by Lemma 4.1.

Propositions 4.2 (and 4.1) imply that MCAP core allocations coincide with the extreme core allocations in BB games. Nevertheless, as we have shown in the end of Section 3, this statement does not hold for assignment games. Characterizing MCAP core allocations in assignment games is still an open problem.

\section{References}

[1] Bondareva, O. 1963. Some applications of linear programming methods to the theory of cooperative games. Problemi Kobernet 10: 119-139. [In Russian]

[2] Diestel, R. 2017. Graph Theory. 5th ed. Springer.

[3] Hammers, H., Klijn, F., Solymosi, T., Tijs, F., Villar, J.P. 2002. Assignment games satisfy the CoMa-property. Games and Behavior 38: 231-239.

[4] Núñez, M., Rafels, C. 2003. Characterization of the extreme core allocations of the assignment game. Games and Economic Behavior 44: 311-331.

[5] Izquierdo, J., Núñez, M., Rafels, C. 2007. A simple procedure to obtain the extreme core allocations of an assignment market. International Journal of Game Theory 36: 17-26.

[6] Shapley, L. 1967. On balanced sets and cores. Naval Research Logistics 14: 453-460.

\footnotetext{
${ }^{4}$ In this subcase, $R\left(\sigma_{k^{*}-1}\right)=\{m\}$ implies that $k^{*}-1=1$, i.e., $k^{*}=2$.
} 
[7] Shapley, L., Shubik, M. 1969. On market games. Journal of Economic Theory 1: 9-25.

[8] Shapley, L., Shubik, M. 1971. The assignment game. I. The core. International Journal of Game Theory 1: 111-130.

[9] Sprumont, Y. 1990. Population monotonic allocation schemes for cooperative games with transferable utility. Games and Economic Behavior 2: 378-394.

[10] Thomson, W. 1983. The fair devision of a fixed supply among a growing population. Mathematics of Operations Research 8: 319-326.

[11] Thomson, W. 1983. Problems of fair division and the egalitarian solution. Journal of Economic Theory 31: 211-226. 